\title{
Les enjeux agricoles et sociétaux de l'eau sur les monts Bamboutos (Cameroun)
}

\author{
Célestin Kaffo ${ }^{1}$ \\ Guillaume Fongang ${ }^{2}$ \\ ${ }^{1}$ Institut national de cartographie \\ BP 157 \\ Yaoundé \\ Cameroun \\ <celkaf2000@yahoo.fr> \\ ${ }^{2}$ Département de vulgarisation agricole \\ et de sociologie rurale Université de Dschang \\ BP 31 \\ Dschang \\ Cameroun \\ <guillaumefongang@yahoo.fr>
}

\begin{abstract}
Résumé
Depuis plus de deux décennies, les enjeux agricoles et sociétaux de l'eau dans les montagnes du Cameroun occidental se sont intensifiés, en raison du développement de l'agriculture intensive marchande de la part des ruraux et migrants de retour, et diversifiés afin de répondre au renforcement de la variabilité climatique inter- et intrasaisonnière. En dépit des efforts des maraîchers et des éleveurs pour adapter leurs pratiques agricoles et pastorales à une diminution de la ressource en eau, les conflits de voisinage se sont multipliés entre ces différents consommateurs. C'est surtout en saison sèche (de décembre à février), période d'étiage des cours d'eau et en cas de sécheresse ponctuelle aggravée que les conflits pour l'accès et l'usage de l'eau s'intensifient. Cette situation s'avère d'autant plus paradoxale que la zone d'étude dispose de fortes potentialités hydrologiques et hydriques et présente un effet orographique marqué de la pluviométrie, avec plus de $2000 \mathrm{~mm}$ de pluies par an sur les versants des monts Bamboutos. Ces conflits peuvent parfois aller jusqu'à la destruction des parcelles maraîchères et la perte de bétail pour les éleveurs peuls ou le décès de certains protagonistes (conflit entre les chefferies de Fongo-Tongo et de Bafou). Pour faire face à ce type de situation, les autorités locales ont développé des stratégies de conciliation selon la nature du conflit et le type de droits revendiqués.
\end{abstract}

Mots clés : Cameroun ; déficit pluviométrique ; production végétale ; système agraire.

Thèmes : eau ; économie et développement rural.

\section{Abstract}

\section{Agricultural and societal importance of water on the Bamboutos mountains (Cameroon)}

For more than two decades, water on the mountains of western Cameroon has become an increasingly high stakes agricultural and societal resource. This situation is due to the development of speculative crops with high economic value by rural and neo-rural populations along with diversification aimed at responding to the reinforcement of inter- and intraseasonal climatic variation. In spite of the efforts of market gardeners and livestock grazers to adapt their production systems to water shortage, neighbourhood conflicts have multiplied between these consumers. It is especially in the dry season (from December to February), a period of low water level in rivers and worsened by specific dryness that the conflicts for the access and the use of water intensify. This situation appears paradoxical since this zone not only has enormous hydrological and hydric potential but equally has the most drained and watered slopes of the region with more than $2000 \mathrm{~mm}$ of rainfall per year on the Bamboutos mountain slopes. These conflicts lead to the destruction by Fulani grazers of goods belonging to market gardeners. As a solution to these recurrent conflicts, the local authorities have developed ruling strategies according to the nature of the conflicts and the rights claimed.

Key words: agrarian systems; Cameroon; plant production; precipitation deficit.

Subjects: economy and rural development; water. 
$L^{\prime}$ analyse des enjeux agricoles et sociétaux sur les montagnes du Cameroun occidental a souvent porté sur la concurrence spatiale entre agriculteurs Bamiléké, pasteurs peuls et mbororo, et éleveurs anglophones (Ngoufo, 1988 ; Dongmo, 1994 ; Boutrais, 1995 ; Fotsing, 1998). Cette pression sur les terres d'altitude est moins imputable au phénomène de colonisation agricole spontanée des ruraux qu'à l'appropriation rapide, précoce et parfois abusive du foncier par les élites (nouveaux " grands " notabilisés ou fonctionnaires absentéistes) par des dons symboliques ou des achats auprès des chefs traditionnels. D'après Le Roy et al., (1996), cette appropriation révèle qu'au-delà du revenu que l'on peut tirer de l'exploitation d'une parcelle de terre, le foncier symbolise un type de pouvoir et de statut social. Depuis le milieu des années 1990, le foncier rural a connu une réelle plus-value en pays bamiléké, sur fond de crise économique, caféière et foncière survenues à la fin des années 1980 (Kuété, 1996 ; Janin, 2000). Le défi est plus que jamais de pouvoir assurer l'alimentation quotidienne des espaces fortement densifiés des "Hautes Terres de l'Ouest " - on relève des densités comprises entre 169 et 1908 hts $/ \mathrm{km}^{2}$ sur 374 km² dans les Bamboutos entre 1976 et 1985 (Fotsing, 1995) - et maintenir les flux d'approvisionnement vivrier en direction des villes camerounaises. De telle sorte que la colonisation des " marges " au sein de chaque chefferie bamiléké est devenue une sorte de "nouvelle frontière " pour les anciens caféiculteurs, les "jeunes" et les " migrants de retour " et les exclus du système foncier traditionnel par suite de partages successoraux (Janin, 1999). Cette dynamique foncière et agricole passe par la colonisation des marges et le développement du maraîchage, jugé plus rentable à l'hectare (Kaffo, 2005). Ce phénomène de "retour à la terre " contribue à accélérer le changement social au sein du ménage comme de l'unité familiale de production et à faire évoluer les rapports entre les sexes et entre les générations (Kaffo, 2005). "Ressource multiusage " (activité agropastorale, irrigation, alimentation des animaux et usage domestique), selon le vocabulaire désormais consacré, la terre et l'eau constituent sur cet espace exigu, une pierre d'achoppement permanent entre agriculteurs " reconvertis ", agriculteurs "de souche" et éleveurs peuls transhumants. Ainsi, le concept de foncier ne se rapporte plus uniquement aux modes d'accès et d'usage du sol ; il est devenu plus englobant et s'étend désormais aux ressources naturelles que porte le sol. Avec cette nouvelle vision, on peut conclure avec Lavigne Delville et al. (1998) que les rapports sociaux sont désormais révélateurs des rapports fonciers. Mais si les conflits de voisinage se multiplient et perdurent (Caron et Torre, 2002), c'est aussi parce que les logiques normatives de résolution des différends ont du mal à cohabiter, aucune ne parvenant à l'emporter définitivement : l'État veut faire prévaloir le fait que "toute terre non immatriculée lui appartient ", tandis que la société locale ne se réfère qu'au droit coutumier qui reconnaît à la chefferie un pouvoir territorial bien établi (Kaffo, 2000). L'étude réalisée s'efforce d'identifier les facteurs de renouvellement des enjeux agricoles et sociétaux de l'eau en zone d'altitude, les types de conflits qui en découlent et leurs mécanismes de règlement.

\section{Méthode et hypothèse}

Cette étude s'appuie sur une enquête par questionnaire auprès d'un échantillon de 75 agriculteurs (anciens employés du Centre d'expérimentation et d'instruction pour la promotion des semences ayant fait 20 ans dans la pratique du maraîchage) dans trois quartiers établis entre 1800 et 2400 m d'altitude (Femmock, Tali et Aghong) et de 25 éleveurs logés au-delà de 2400 m d'altitude des Bamboutos. Ce massif constitue un vaste volcan bouclier, à la caldeira semielliptique résiduelle occupant le Nord Ouest du plateau Bamiléké, qui culmine à $2740 \mathrm{~m}$ au mont Méléta. Bien arrosés, les nombreux cours d'eau sont répartis en cinq bassins hydrographiques (figure 1) à l'aspect radiaire et centrifuge. Le bassin de la Ménoua, qui concerne plus particulièrement notre étude correspond au versant méridional aux potentialités agronomiques favorables au développement de l'agriculture maraîchère marchande. Les observations de terrain ont permis d'identifier des points où des conflits opposent régulièrement les éleveurs aux agriculteurs, d'une part, et ces derniers entre eux d'autre part. De telles zones ont donc été choisies pour servir de sites d'enquêtes.

Deux hypothèses sous-tendent cette recherche :

- les variations climatiques récentes sont partiellement à l'origine de la réorientation culturale et de la réorganisation spatiale des terroirs bamiléké;
- les évolutions récentes des systèmes de culture (spécialisation maraîchère) dans cet espace saturé, aux fortes potentialités hydrauliques, préfigurent certaines formes de confrontation difficile à réguler. Le principal objectif de cette étude visait à mieux appréhender les enjeux agricoles et sociétaux de l'eau dans un milieu "agro-écologique très favorable".

\section{Facteurs \\ de renforcement des enjeux agricoles et fonciers d'altitude}

\section{Concurrence pour la légitimation du foncier d'altitude}

Les terres de réserves, notamment les terres d'altitude dans les chefferies bamiléké intégrant la montagne dans leur finage, ont été sollicitées dès l'expansion du café. À la faveur d'une colonisation agricole rapide les agriculteurs anglophones, remontant de la cuvette de Mamfé, et les habitants de la chefferie de Bafou, partant à l'assaut de ce qu'ils considèrent être leur réserve foncière d'altitude, ont fortement dégradé, par le feu, par la machette et la houe, la végétation naturelle des Bamboutos à l'exception de quelques îlots forestiers (Morin, 1996). Dans ce milieu, la concurrence spatiale a donné naissance à des formes d'occupation diverses. Les agriculteurs, pour occuper l'espace pastoral, peuvent enclore les pâturages - où ils en viennent à pratiquer un élevage "de circonstance" - qu'ils mettront en culture peu de temps après. Les éleveurs peuls, pour leur part, s'efforcent de maintenir des troupeaux transhumants tout en s'essayant à la culture maraîchère. Mais ces tentatives restent fragiles, systématiquement découragées par les agriculteurs bamiléké qui développent différentes stratégies - vols successifs des bêtes par exemple - pour déloger les éleveurs peuls des sommets, à tel point que les éleveurs peuls ne pratiquant aucune activité agricole complémentaire, ont été progressivement refoulés au-delà de $2400 \mathrm{~m}$ d'altitude. La ligne de démarcation entre l'espace pastoral et l'espace agricole, fixée autour de $2000 \mathrm{~m}$ d'altitude, n'existe plus. À certains égards, la compétition pour l'accès à l'eau est le prolongement du conflit foncier d'altitude, plus ancien, si l'on considère que la sécurisa- 


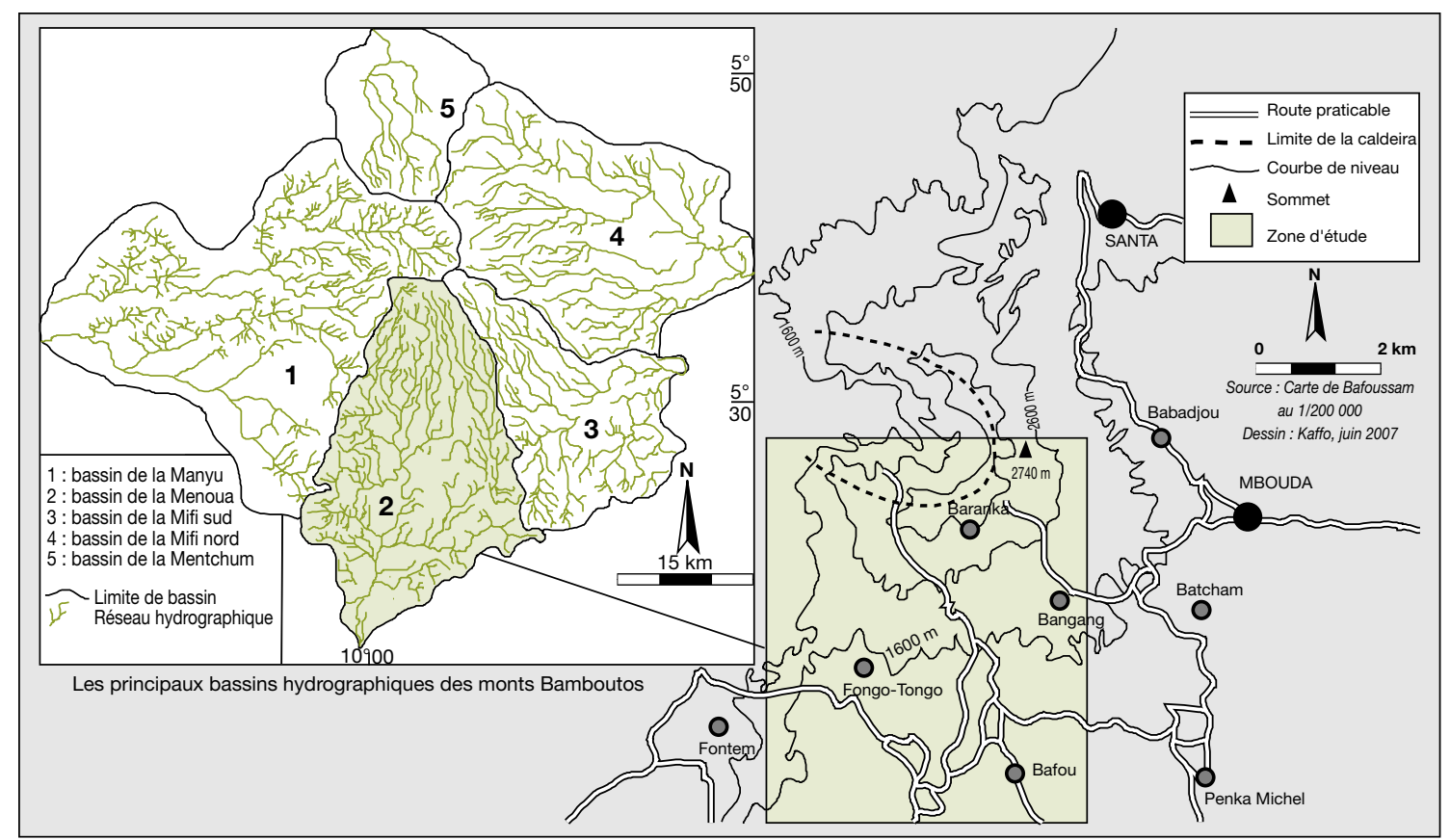

Figure 1. Localisation de la zone d'étude.

Figure. 1. Location of the study area.

tion foncière passe par l'affirmation du droit de propriété sur la gestion des ressources naturelles (Le Roy et al., 1996). Le maraîchage se diffuse donc dans les espaces interstitiels des terroirs de Hautes Terres de l'Ouest du Cameroun, dans un contexte de lutte pour la légitimation de la terre par les agriculteurs au détriment des éleveurs qui ont longtemps occupé l'espace montagnard. À l'exception des promoteurs privés à l'instar de la ferme EPA (Élevage promotion africaine), les agriculteurs ne disposent pas de bétail.

\section{Diversification agricole et essor des cultures maraîchères}

En une seule décennie, on est ainsi passé d'une polyculture familiale intensive en intrants à base caféière à des systèmes vivriers complexes à forte intensification en facteur travail, y compris dans les " marges " peu valorisées où l'on pratiquait essentiellement une agriculture extensive et saisonnière. Sur fond de repli étatique et de libéralisation des filières agricoles, la caféiculture a été remplacée par le marâchage et les cultures vivrières; les zones marginales des altitudes supérieures sont prises d'assaut pour des cultures tempérées à court cycle végétatif (Grangeret Owona,
1994). Ce phénomène s'explique par l'appui de plusieurs structures d'encadrement. Dès la création du Centre d'expérimentation et d'instruction pour la promotion des semences (CEIPS) en 1973 sur le versant occidental des monts Bamboutos, des stages de formation maraîchère ont été organisés. En 1978, le CEIPS est devenu un centre de multiplication de semences avec un effet positif sur la diffusion et la diversification des cultures maraîchères. Peu après, en 1980, le "Projet Hauts Plateaux de l'ouest " (PHPO), relayé en 1984 par le Projet de développement rural de la province de l'ouest (PDRPO) jusqu'en 1993, va considérablement accélérer cette vulgarisation par des actions d'aménagement des bas-fonds inexploités (Grangeret Owona, 1995). Plusieurs autres sociétés soustraitantes dans les fermes agroindustrielles installées à Babadjou ont participé efficacement à la diffusion du marâ̂chage à l'ouest du Cameroun (Kaffo, 2005) par la diffusion de techniques culturales modernisées : nouvelles associations culturales, micro-irrigation gravitaire, utilisation d'intrants agricoles. Cet essor est encore accentué par l'augmentation de la demande alimentaire urbaine et les perspectives de gains monétaires immédiats et réguliers que cette agriculture nourricière et marchande permet. Ces exigences ont conduit à de nombreuses innovations : l'in- tensification et la densification des cultures, la modification du calendrier agricole classique qui passe d'une à trois campagnes agricoles par an (Kuété et al., 2003). De plus en plus, les décisions d'achat ou de location de parcelles situées dans ces espaces "pionniers", à forte valeur agroécologique (sols hydromorphes de basfonds ou sommets de versants volcaniques), ne prennent plus seulement en compte la superficie, mais aussi certains avantages comparatifs : accès à l'eau en saison sèche, présence d'une route à proximité. Ainsi, entre 1990 et 2005, les superficies cultivées ont augmenté de plus de 80 \%, entraînant du même coup un déplacement du front pionnier de 1600 à 2400 m d'altitude (figure 2).

\section{Facteurs déclencheurs de la raréfaction \\ de l'eau}

\section{Pression des activités sur une ressource en régression}

Les besoins en eau des activités agropastorales en saison sèche incitent les actifs à migrer vers les bords des cours d'eau et 


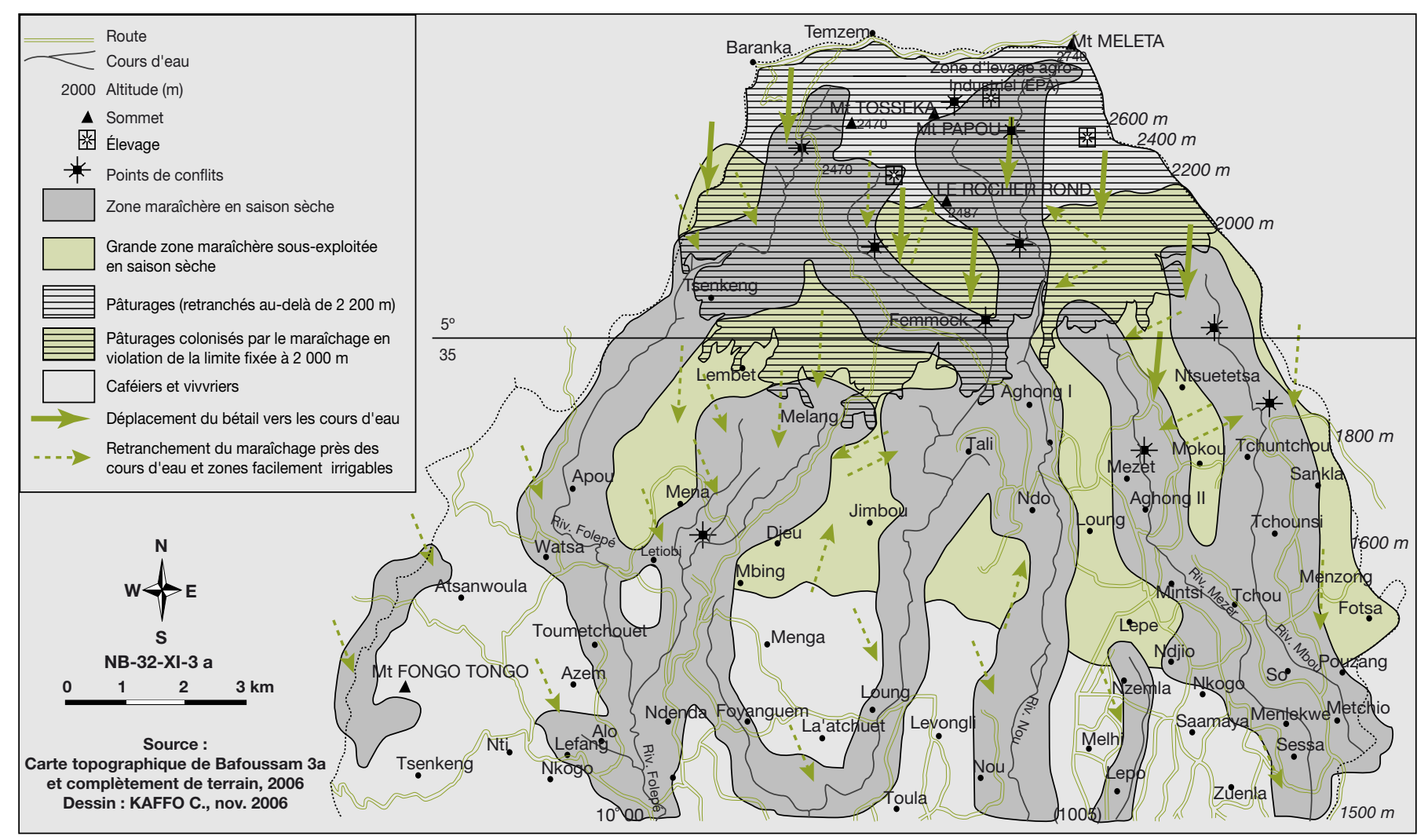

Figure 2. Déficit hydrique et gestion de l'espace sur le versant méridional des monts Bamboutos.

Figure 2. Water shortage and space management on the southern slope of the Bamboutos Mountain.

les vallées hydromorphes également convoités par les éleveurs qui descendent des " hauts " (figure 2). Peu à peu, l'eau est devenue - et ce n'est sans doute pas le seul paradoxe dans cet espace bien arrosé - au même titre que la valeur économique des cultures de contre-saison, un élément déterminant de la marchandisation des terres des bas-fonds. Dans les grandes zones de tradition maraîchère telles que les chefferies de Bafou, de Fongo-Tongo, de Bansoa dans la Ménoua; les chefferies de Babadjou, de Bangang, de Galim, de Bamedjing dans les Bamboutos (Dongmo, 1983 ; Champaud, 1983), de Foumbot en pays Bamoun et de Santa dans la Mézam (Kaffo, 2005), ces éléments ont signé l'apparition de nouvelles identités spatiales et territoriales en lieu et place de la précédente hiérarchie " haut-bas " qui caractérisait la géopolitique du terroir Bamiléké. Ainsi, si les points hauts et culminants sont devenus des sites recherchés par de nombreuses élites en mal de reconnaissance symbolique et ostentatoire pour la construction de vastes villas à étages au style parfois tapageur, les bas-fonds aux sols hydromorphes sont de plus en plus convoités par les agriculteurs en saison sèche. Cela explique la forte concurrence

pour l'accès à ces parcelles des bas-fonds et à l'eau qu'elles possèdent.

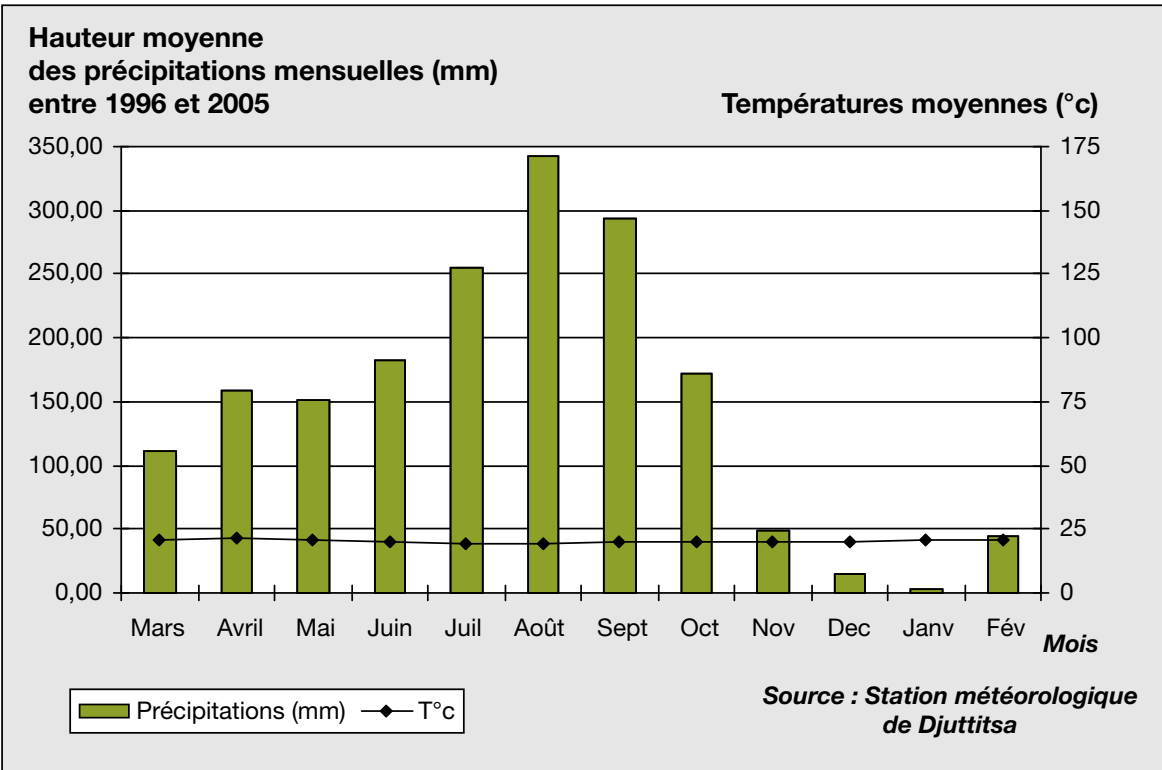

Figure 3. Données climatiques de Djuttitsa de 1996 à 2005.

Figure 3. Climatic data of Djuttitsa from 1996 to 2005. 


\section{Tableau 1. Quantité d'eau indispensable par cycle cultural.}

Table 1. Available water quantity per crop cycle.

\begin{tabular}{llll|ll}
\hline Cultures & \multicolumn{2}{c|}{$\begin{array}{c}\text { Quantité d'eau indispensable } \\
\text { pour la croissance des plantes par cycle (cm) }\end{array}$} & $\begin{array}{c}\text { Quantité d'eau indispensable } \\
\text { pour la croissance des plantes de 3 } \mathbf{3}^{\mathbf{c}} \mathbf{c y c l e} \text { (cm) }\end{array}$ \\
\cline { 2 - 6 } & $\begin{array}{l}\text { Premier cycle } \\
\text { (mars-juin) }\end{array}$ & $\begin{array}{c}\text { Deuxième cycle } \\
\text { (juillet-octobre) }\end{array}$ & $\begin{array}{l}\text { Troisième cycle } \\
\text { (novembre-février) }\end{array}$ & Disponibilité hydrique Déficit hydrique \\
\hline Chou & 65 & 91 & 30 & 12 & -18 \\
Pomme de terre & 65 & 72,9 & 50 & 30,6 & $-19,4$ \\
Carotte & 37,7 & 72,9 & 40 & 20,2 & $-19,8$ \\
Poireau (bottes) & 84,4 & 130 & $40-60$ & $19-31$ & $-21-29$
\end{tabular}

\section{Variabilité climatique inter- et intrasaisonnière}

L'analyse des données pluviométriques observées sur la période 1996-2005 (figure 3), pour la station météorologique de Djuttitsa, montre un climat de type subéquatorial de mousson à deux saisons avec une influence prépondérante de la montagne. Les mois de décembre, janvier et février présentent d'importants déficits hydrologiques, évalués à environ $140 \mathrm{~mm}$. La baisse considérable de l'easily used reserve (EUR) à partir de novembre, explique le déficit hydrique le plus bas du versant sud du massif observé en décembre avec des températures élevées de l'ordre de $21^{\circ} \mathrm{C}$ (Njiosseu Tanko, 1997). Toutefois, avec les premières pluies de mars et d'avril, l'EUR se reconstitue rapidement. Le déterminisme climatique influence doublement l'activité agricole. Non seulement le découpage des saisons - saison sèche et saison des pluies - oriente plus ou moins le calendrier agricole, mais aussi les paysans font face à des séquences sèches, qui sur- viennent parfois pendant les mois de mars, avril, mai et octobre, suite à l'arrivée tardive ou l'arrêt brutal des pluies. Lorsque ces épisodes climatiques cö̈ncident avec les périodes importantes de la croissance de la plante, les agriculteurs ont obligatoirement recours à une demande supplémentaire en eau.

\section{Indicateurs spatiaux du déficit hydrique et orientations}

paysannes dans la production spatiale

\section{Indicateurs spatiaux du déficit hydrique}

La raréfaction de l'eau nécessaire à la croissance des plantes de troisième cycle (tableau 1), l'assèchement des cours d'eau, le flétrissement des plantes et la corvée pour l'eau de consommation sur le plateau figurent parmi les indicateurs spatiaux de déficit hydrique (figure 4). Les agriculteurs attestent que depuis environ 20 ans la baisse du volume des eaux est perceptible mais désigne la variabilité climatique inter- et intrasaisonnière ainsi que la déforestation comme responsables de la baisse de la nappe phréatique dans cet espace à fortes potentialités hydrographiques et hydriques. Des études sur la variabilité climatique et son impact sur l'alimentation des aquifères souterraines en Côte-d'Ivoire parviennent à des résultats probants : baisse notable, de plus en plus fréquente, du débit des cours d'eau et du niveau des nappes phréatiques allant jusqu'à l'assèchement (Brou et al., 2005). Par ailleurs, les mesures faites par Monka Njotu (2002), sur une période de six mois sur le principal cours d'eau à $2300 \mathrm{~m}$ d'altitude, illustrent cette situation (tableau 2).
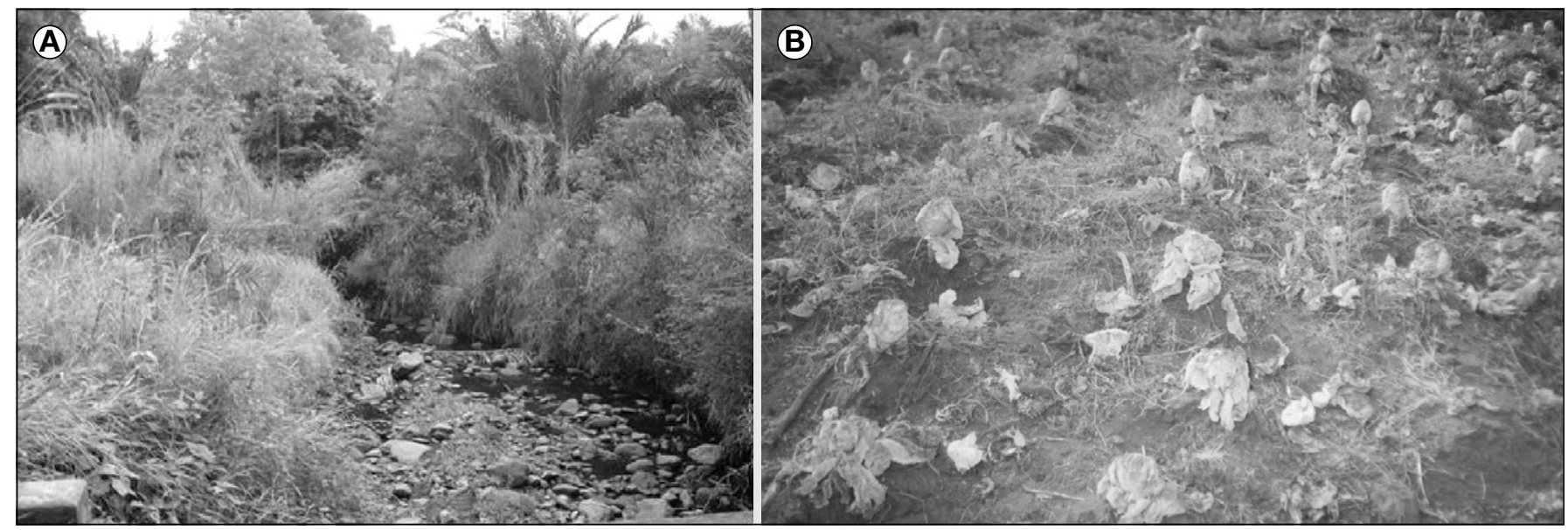

Figure 4. Les indicateurs spatiaux du déficit hydrique.

Figure 4. Spatial indicators of water shortage.

A) assèchement d'un cours d'eau ; B) flétrissement des plants de chou dû à l'insuffisance de l'eau. 
Tableau 2. Éléments d'identification de la baisse du débit d'un cours d'eau.

Table 2. Elements identifying low flow debits.

\begin{tabular}{lcccccc}
\hline Éléments de mesure & Novembre & Décembre & Janvier & Février & Mars & Avril \\
\hline Largeur du cours d'eau $(\mathrm{cm})$ & 4,6 & 3,3 & 3 & 3,5 & 6 & 7 \\
Profondeur du cours d'eau (cm) & 3,5 & 2,6 & 3,6 & 3,8 & 5 & 5,8 \\
Vitesse moyenne d'écoulement $\left(\mathrm{cm}^{3} / \mathrm{s}\right)$ & 22,3 & 15,4 & 18,3 & 20,3 & 38,8 & 40,2 \\
Débit d'écoulement $\left(\mathrm{cm}^{3}\right)$ & 202,2 & 156 & 162,3 & 183,4 & 219,3 & 282,8 \\
\hline
\end{tabular}

\section{Adaptation de l'irrigation comme solution au déficit hydrique}

Les nouvelles contraintes climatiques et le déficit hydrique ont conduit les agriculteurs à développer une pluralité de réponses adaptatives telles que la diversification et la multiplication des dispositifs d'irrigation. L'irrigation gravitaire à la raie: le maraîcher pratique une simple dérivation d'un cours d'eau jusqu'à sa parcelle. L'eau ainsi déviée circule dans les sillons, s'infiltre et atteint directement le système racinaire de la plante. Cette forme a comme variante "l'irrigation à l'assiette " pratiquée dans les parcelles à topographie plane (plaine de Darmagnac à Babadjou). Les maraîchers, à l'aide d'une assiette, versent manuellement l'eau qui coule dans les sillons entre les cultures en billons. Avec l'irrigation gravitaire par aspersion, le maraîcher à l'aide d'un tuyau de $11 \mathrm{~mm}$ de diamètre, placé sur un tourniquet, arrose les plantes. Enfin, avec une motopompe et des tuyaux PVC de 63 ou de $100 \mathrm{~mm}$ de diamètre, le maraîcher fait remonter de l'eau pour irriguer sa parcelle. L'engouement des agriculteurs pour les différentes techniques d'irrigation va de pair avec la bonne fonctionnalité sociétale des systèmes informels d'irrigation sur les Hautes Terres de l'Ouest (Teheubeng, 1999; Kamga, 2002). Toutefois, la diversité des usages de l'eau et la hausse de la demande tendent à complexifier sa gestion.

\section{Émergence des conflits} face aux enjeux d'usagers divers de l'eau

Coulibaly (2006) souligne que les conflits relatifs à la gestion des ressources naturelles (terre, eau, forêt, etc.) deviennent, depuis quelques années, de plus en plus fréquents et difficiles à gérer en milieu rural humide. Très souvent limités spatialement, les terroirs maraîchers de saison sèche sont d'autant plus recherchés qu'ils sont rémunérateurs, et que leurs productions pallient pour partie les revenus des produits vendus en saison de pluie et la baisse concomitante des budgets domestiques (Fromageot, 2006). L'eau génère donc des situations de compétition, à l'origine des conflits qui opposent les éleveurs aux agriculteurs ou ces derniers entre eux; acteurs aux intérêts divergents, mais souvent obligés de partager le même point d'eau.

\section{Conflits entre agriculteurs}

La compétition pour l'accès et la maîtrise de l'eau se matérialise parfois, sur le terrain, par la concentration de nombreux tuyaux d'irrigation (plus d'une vingtaine) appartenant à plusieurs agriculteurs autour d'un seul point d'eau (figure 5). Dans cette configuration, certains agriculteurs peuvent même procéder au détournement des tuyaux ou à leur vol. Ces actes sont à l'origine des disputes et d'inimitiés entre les agriculteurs et se soldent par la destruction systématique des récoltes et des tuyaux du mis en cause.

\section{Conflits entre agriculteurs et éleveurs}

Au fil des années souligne Boutrais (1995), l'extension générale des cultures et l'hostilité croissante à l'égard des éleveurs peuls de la part des agriculteurs Bamiléké ont progressivement gommé les repères pastoraux. Le non-respect de la ligne de démarcation entre l'espace agricole et pastorale établie à $2000 \mathrm{~m}$ d'altitude, les mouvements de transhumance qu'effectuent les éleveurs et le déplacement des agriculteurs à la recherche de l'eau occasionnent des conflits parfois ouverts (Coulibaly, 2006). Pour marquer leur hostilité à l'occupation des pâturages, les éleveurs laissent leurs animaux s'introduire dans les exploitations agricoles ou s'abreuver dans les retenues d'eau aménagées par les agriculteurs. Cet usage entraîne de fréquentes destructions des clôtures et des cultures (Fromageot, 2006). Ces confrontations individuelles se sont multipliées dans les années 1990 avec l'essor du maraîchage de contresaison. Elles se sont parfois transformées en conflits communautaires (maraîchers, agriculteurs, pasteurs). Tsalefac (1994) souligne que de 1982 à 1985, plus d'une

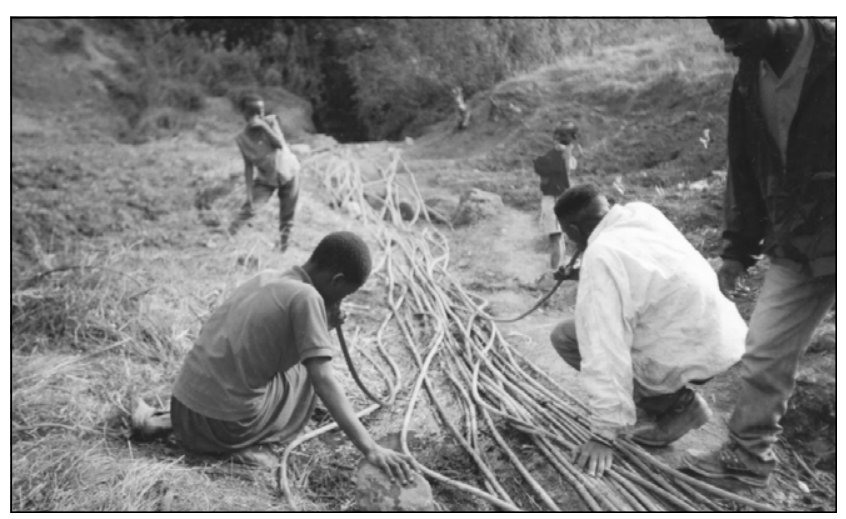

Figure 5. Compétition pour l'eau en saison sèche.

Figure 5. Water competition during the dry season. 
centaine de conflits agropastoraux ont été portés à l'attention du Chef de poste agricole (CPA) de Djuttitsa. Entre 1995 et 2005, une trentaine de conflits - ayant pour objet la destruction des enclos autour des surfaces cultivées, des dispositifs d'irrigation et l'incendie des sacs de fertilisants appartenant aux agriculteurs, l'incendie des camps des bouviers, le massacre systématique, les vols et l'empoisonnement des bêtes (en déversant des produits toxiques dans les points où s'abreuvent les boufs) par les agriculteurs (figure 6) - ont aussi été soumis à l'arbitrage des chefs de quartier Tali, Femmock et du CPA de Djuttitsa. Par ces tensions, les acteurs reposent la question de légitimité ou de droit de propriété des réserves des terres d'altitude.

\section{Difficile accès à l'eau potable domestique}

La surconsommation de l'eau en altitude est à l'origine de graves pénuries en contrebas sur le plateau de sorte que les adductions d'eau moderne qui fonctionnent par gravitation sont parfois vides (figure 6A). La collecte de l'eau de boisson devient pour des ménages en aval, très problématique sans compter les risques de contamination potentielle à cause de surdosages en intrants agricoles. En réponse, les habitants des piémonts sont parfois obligés d'aller rompre les digues sur la montagne.

\section{Mécanismes}

\section{de règlement des conflits}

\section{Conflits entre agriculteurs}

Le règlement des conflits entre agriculteurs repose sur des logiques et des stratégies spécifiques telles que le savoir-faire de certains agriculteurs expérimentés (anciens ouvriers du CEIPS, agents en service ou retraités des services des ministères de l'Agriculture, de l'Élevage) et des autorités coutumières qui procèdent, chaque fois, à une réorganisation du système de gestion de l'eau par rive. Ainsi, les agriculteurs utilisant le même point d'eau se regroupent et élaborent un calendrier d'utilisation de l'eau. Autour d'un même point d'eau, les agriculteurs conviennent d'une répartition par tranches horaires sous le contrôle des chefs de blocs désignés par les chefs de quartier et le CPA de Djuttitsa. Faute de quoi, chaque contrevenant est sommé de payer une amende de 10000 francs CFA par tranche d'une heure et cette sanction peut aller jusqu'à la privation totale de l'irrigation. En amont de la zone maraîchère d'altitude, les agriculteurs construisent de petits réservoirs le long des cours d'eau grâce auxquels l'eau est facilement dirigée dans les parcelles cultivées.

\section{Conflits entre agriculteurs et éleveurs}

Entre éleveurs et agriculteurs, les revendications portent plutôt sur les dommages causés par le bétail. À ce sujet, Coulibaly (2006) souligne qu'" elles relèvent, par conséquent, de conflits d'intérêts économiques opposant des acteurs de différentes catégories professionnelles: agriculteurs et éleveurs". Il est à rappeler que les techniques d'irrigation, mises au point par les agriculteurs pour pallier le déficit hydrique, ont permis d'étendre les cultu-

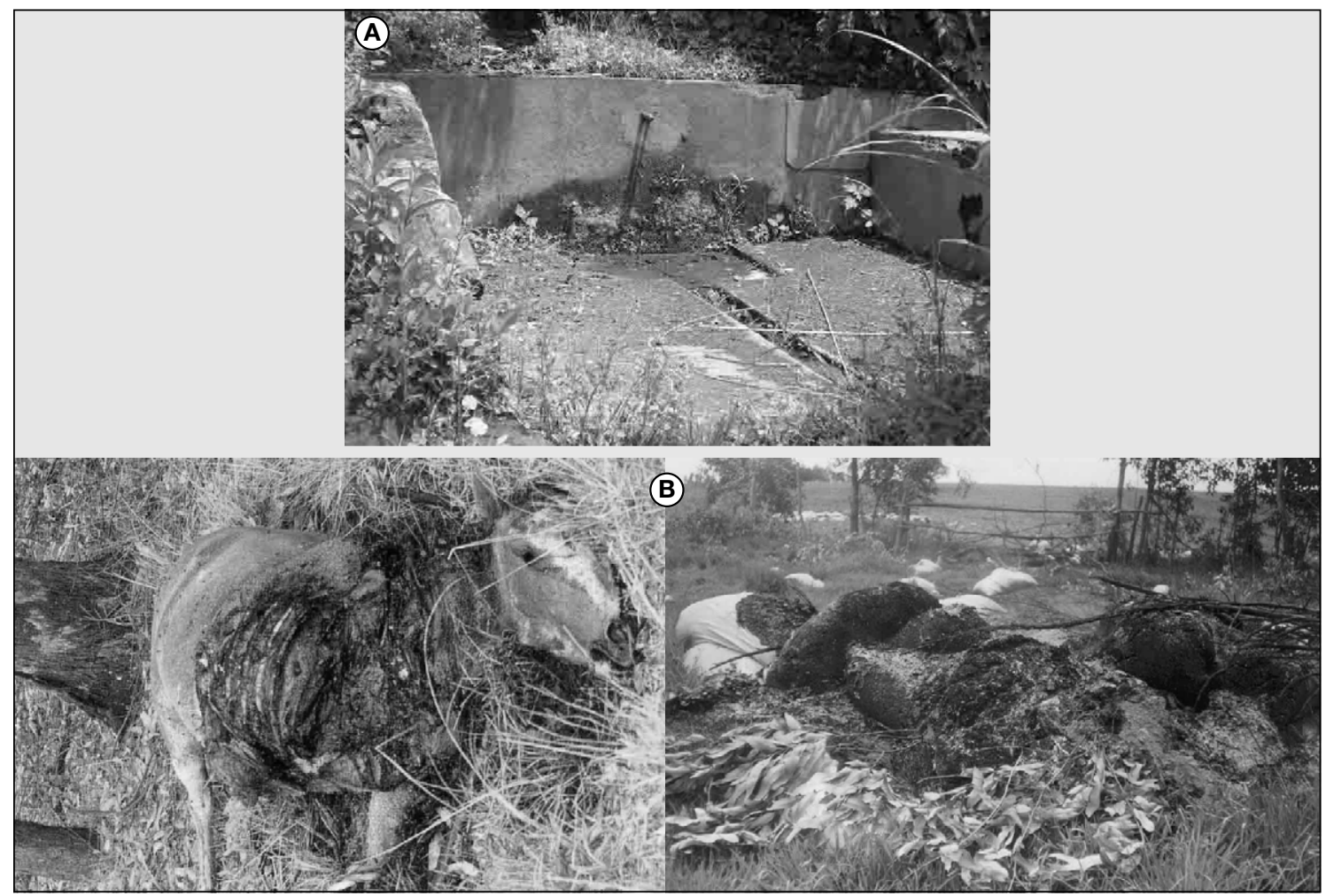

Figure 6. Les conséquences du déficit hydrique.

Figure 6. Consequences of the water shortage.

A) Impact du déficit hydrique sur l'approvisionnement en eau potable ; B) les dégâts du conflit entre maraîchers et éleveurs pour la gestion de l'eau et de l'espace. 
res maraîchères sur les espaces jadis impropres à cette activité, et réservés au pâturage. Ainsi, l'offensive du maraîchage sur le massif réduit désormais les pâturages à des portions confinées et très fragmentaires (figure 2). Pour calmer les tensions entre éleveurs et agriculteurs, les autorités administratives ont, en 1984, fixé de manière quelque peu arbitraire, à 2000 m d'altitude, une limite entre espace agricole et espace pastoral. Cette disposition vient compléter les mesures prises par le décret $n^{\circ} 78 / 263$ du 03 juillet 1978. Malheureusement, ces mesures n'ont ni freiné l'invasion progressive des pâturages par les agriculteurs ni résolu de façon définitive les différends. De nos jours, les chefs de quartiers ont la charge de régler les conflits au cas par cas ou de les porter devant une commission regroupant les responsables des ministères de l'Agriculture, de l'Élevage et du sous-préfet de la localité ou encore devant les juridictions compétentes au cas où les parties ne respecteraient pas les résolutions proposées. Les membres de la commission, après constat, demandent aux protagonistes de négocier entre eux la somme à payer. D'une manière générale, les résolutions prises préconisent aux parties de respecter leurs domaines respectifs d'activité. Les éleveurs ont la charge de surveiller leurs animaux ou d'éviter de les introduire de façon délibérée dans les exploitations agricoles ou dans les points de retenue d'eau aménagés par les agriculteurs. La difficile gestion de l'espace et de l'eau, résultat des conflits divers, expose les ressources de ce milieu à une exploitation intense et peu judicieuse (Bethemont, 1986). On est donc loin d'un compromis qui permettrait aux uns et aux autres de tirer profit de cet espace et qui engagerait les différents acteurs à une gestion durable des ressources naturelles.

Les conflits opposant agriculteurs et éleveurs et les conflits entre agriculteurs pour le contrôle des ressources naturelles conduisent à la mise en place de dispositifs territoriaux que Fongang (2004) définit comme toute dynamique impliquant les acteurs appartenant à un même territoire, engagé dans un processus de définition à leur échelle de politiques ou de choix concernant le présent et/ou l'avenir du territoire.

\section{Perdants et gagnants de la gestion de l'eau et du foncier}

Les agriculteurs sont généralement les "gagnants" de la gestion de l'eau et de la conquête foncière contrairement aux éleveurs peuls dont les pâturages sont réduits aux confins des fortes pentes (figure 2). Pour appuyer sa plainte contre les éleveurs et obtenir gain de cause, l'agriculteur a tôt fait de clamer que "le champ n'a pas de pied, c'est l'animal qui se déplace "(Coulibaly, 2006) même s'il est parfois l'auteur du conflit. Dans l'ensemble, les arbitrages réels des différents conflits s'effectuent par un arrangement à l'amiable assorti d'amendes proportionnelles aux dégâts causés.

\section{Conclusion}

Les effets de la variabilité climatique interet intrasaisonnière ont renforcé la dépendance et les difficultés d'approvisionnement en eau en période d'étiage des cours d'eau dans les monts Bamboutos. La forte demande en eau, l'intense mise en valeur de l'espace et le déboisement intégral des formations arborées d'altitude depuis la fin des années 1985 ont progressivement finit par assécher les nappes phréatiques et les cours d'eau autrefois permanents. Pour faire face au manque d'eau, les paysans ont peu à peu fait évoluer leurs pratiques renforçant, par la même occasion, la dimension agricole et sociétale de l'eau. Ainsi, ont-ils, par exemple, été obligés d'utiliser des variétés à cycle court et moins consommatrices en eau, de coloniser les zones humides ou facilement irrigables (bas de pente, berges des cours d'eau, bas-fonds) et de développer des techniques d'irrigation. La mise en valeur de ces espaces montre que la pratique d'une agriculture marchande, fondée sur un système de production extensif et exigeant en eau, entraîne un bouleversement des modes de gestion et des représentations sociales. La course à l'eau et au foncier par les agriculteurs et les éleveurs engendre des conflits qui reposent la question de légitimité ou de droit de propriété des réserves des terres d'altitude. Les techniques d'accès à l'eau en altitude rendent difficiles l'accès des populations des piémonts à l'eau potable domestique. Face à la multiplicité des conflits, les autorités locales mettent sur pied certains mécanismes de résolution. Mais, les agriculteurs dans leur conquête permanente (mise en valeur des bas-fonds et surconsommation de l'eau) semblent peu préoccupés par la sauvegarde de l'environnement (destruction des raphiales), mettant ainsi en danger les écosystèmes d'un milieu fragile.

\section{Références}

Bethemont J. Acteurs et stratégies de I'eau dans la vallée du Sénégal. Rev Geogr Lyon 1986 : 63-78.

Boutrais J. Hautes Terres d'élevage du Cameroun. Paris: Orstom éditions, 1995.

Brou Yao T, Akindès F, Bigot $S$. La variabilité climatique en Côte-d'Ivoire: entre perceptions sociales et réponses agricoles. Cah Agric $2005 ; 14: 533-40$.

Caron A, Torre A. Les conflits d'usages dans les espaces ruraux "Une analyse économique ". Sci Soc 2002 ; 57 : 95-113.

Champaud J. Les relations et villes campagnes du Cameroun de l'Ouest. Paris: Orstom éditions, 1983.

Coulibaly A. Gestion des conflits fonciers dans le Nord ivoirien Droits, autorités et procédures de règlement des conflits. Colloque international "Les frontières de la question foncière ", Montpellier, 2006. (À paraître).

Dongmo D. Les cultures maraîchères dans la province de l'Ouest: production et commercialisation. Thèse de doctorat de troisième cycle, université de Yaoundé, 1983.

Dongmo JL. Conflit agriculteurs-éleveurs pour la terre sur le versant méridional des mont Bamboutos (Ouest-Cameroun). Savanisation process in tropical. Africa (Lond) 1994; II: 107-20.

Fongang G. Dynamiques des dispositifs territoriaux: le cas du dispositif agri-environnemental en Corse. Mémoire de DEA MSC, Ina PG - Inra, 2004.

Fotsing JM. Compétition foncière et stratégies d'occupation des terres en pays Bamiléké (Cameroun). Terre, terroir, les tensions foncières. Paris : Orstom éditions, 1995.

Fotsing JM. Problèmes fonciers et élevage bovin en pays bamiléké : exemple du nord de Bafou (Ouest Cameroun). Cah Rech Dev 1998; $20: 45-52$

Fromageot A. Agriculture et conflits en Côted'lvoire: terroirs maraîchers, territoires disputés. Géoconfluences, 2006.

http://geoconfluences.ens-Ish.fr/doc/etpays/ Afsubsah/AfsubsahScient3.htm.

Grangeret Owona I. De la crise à la dévaluation du FCFA: évolution de l'agriculture intensive familiale bamiléké. Cah Agric 1995 ; 4 : 45-51.

Grangeret Owona I. Les nouvelles pratiques des exploitants agricoles bamiléké sous l'effet des ruptures anciennes et de la conjoncture actuelle. Le village camerounais à l'heure de I'ajustement. Paris: Karthala, 1994.

Janin P. Gestion patrimoniale et sécurisation foncière en économie de plantation à I'heure des ajustements. Rev Geogr Cameroun 2000; 14 : 79-107.

Janin P. L'avenir des planteurs camerounais. Paris: Karthala, 1999.

Kaffo C. Cultures maraîchères dans les montagnes du Cameroun occidental. Cah Agric 2005 14 : 517-24.

Kaffo C. Développement du maraîchage d'altitude et marché foncier dans les monts Bamboutos. Déprise caféière et mutations socioéconomiques sur les hautes terres de l'Ouest Cameroun. Geodoc 2000 ; 51 : 24-42.

Kamga A. Crise économique, retour des migrants, et évolution du système agraire sur le versant oriental et méridional des monts Bamboutos (Ouest-Cameroun). Thèse de doctorat 
d'études rurales, université de ToulouseLe-Mirail, 2002.

Kuété M, Dzalla C, Yemmafouo A. Les montagnes du Cameroun face aux nouveaux enjeux de développement de la sous-région Afrique centrale. Crises et mutations des agricultures de montagne. Colloque international en hommage au Pr. C. Mignon. Clermont-Ferrand : Ceremac; Presses universitaires Blaise-Pascal 2003.

Kuété M. Les enjeux des cultures de rentes au Cameroun: l'exemple de la caféiculture. Dschang (Cameroun): Dschang University Press, 1996.

Lavigne-Delville P. Quelles politiques foncières pour I'Afrique rurale?. Paris: Karthala, 1998.
Le Roy E, Karsenty A, Bertrand A. La sécurisation foncière en Afrique: pour une gestion viable des ressources renouvelables. Paris: Karthala, 1996.

Monka Njotu J. A geographical analysis of water problems in agriculture: case of market gar dening on the southern slopes of the Bambou tos Mountain (west-Cameroon). Mémoire de maîtrise de géographie, université de Dschang (Cameroun), 2002.

Morin S. Le haut et le bas : signatures sociales paysages et évolution des milieux dans les montagnes d'Afrique centrale (Cameroun e Tchad). Pays enclavés, $\mathrm{n}^{\circ} 8$. Talence: Presses universitaires de Bordeaux, 1996.

Ngoufo R. Les monts Bamboutos: environnement et utilisation de l'espace. Thèse de doctorat $3^{\mathrm{e}}$ cycle, université de Yaoundé-I, 1988.
Njiosseu Tanko R. Évaluation des potentialités hydrologiques du bassin de la Ménoua, versant méridional des Bamboutos. Mémoire de maîtrise de géologie, université de Dschang (Cameroun), 1997.

Teheubeng A. Évaluation de la performance d'un système d'irrigation de surface à petite échelle sur les hautes terres de l'Ouest. Thèse de Master of Science de gestion de I'eau, université de Dschang (Cameroun), 1999.

Tsalefac M. Crises climatiques récentes et bouleversements du système économique traditionnel à Fongo-Tongo (Monts Bamboutos, $\mathrm{Ca}$ meroun). Savanisation process in tropical. Africa (Lond) 1994 ; II : 95-106. 